\title{
Macroscopic coherence effects in a mesoscopic system: Weak localization of thin silver films
}

\author{
A. D. Beyer, ${ }^{a)}$ M. Koesters, K. G. Libbrecht, and E. D. Black \\ Department of Physics, California Institute of Technology, Pasadena, California 91125
}

(Received 9 March 2005; accepted 29 July 2005)

\begin{abstract}
We present an advanced undergraduate experiment on weak localization in thin silver films with a thickness between 60-200 , a mesoscopic length scale. At low temperatures, the inelastic dephasing length for electrons exceeds the film thickness, and the film becomes quasi-two-dimensional. In this limit, theory predicts corrections to the Drude conductivity due to the coherent interference between the wave functions of the conducting electrons, a macroscopically observable effect known as weak localization. This correction can be destroyed by the application of a magnetic field, and the resulting magnetoresistance curve provides information about electron transport in the film. (C) 2005 American Association of Physics Teachers.
\end{abstract}

[DOI: $10.1119 / 1.2049284]$

\section{INTRODUCTION}

One of the many challenges in a senior level laboratory course is to simulate the research environment. The laboratories generally should be geared toward helping students transition from performing prepackaged experiments to more independent experiments. Experiments of this sort need to be designed so that motivated students can succeed in a term or semester. With these goals in mind, we have designed an experiment for students to observe the phenomena of weak localization.

Weak localization is a macroscopically observable consequence of the quantum mechanical behavior of electrons. Electrons begin to localize around impurities at low temperatures because of self-interference. As the electrons scatter off impurities, certain paths of the partially scattered electron waves add together to localize the electrons. This prelocalization effect is called weak localization and alters the bulk behavior of the electrons.

The bulk property that most clearly is evidence for weak localization is the magnetoconductance, which is best observed in thin film samples at low temperatures (accessible with liquid helium). The magnetoconductance is a small correction to the bulk conductance of a sample, theoretically on the order of $10^{-4}$ of the bulk conductance. In this laboratory, we discuss how samples are made and how the magnetoconductance is measured.

One of the goals of this laboratory is to teach students techniques for measuring small signals. Measuring small signals is a common occurrence in condensed matter experiments and for experimental physicists in general. In our experiment, the magnetoresistive signal we want to measure is swamped by the bulk resistance of the sample. To magnify this weak signal, we use a resistance bridge. The idea is simple, null out the large resistance signal due to the bulk conductance and amplify the remaining signal due to the magnetoresistance. We also review sources of noise that can arise in the bridge, such as ground loops, and how to design the bridge to eliminate them.

Typical student results and a brief interpretation of these results are then discussed. We find that for the $\mathrm{Ag}$ thin film samples made in our laboratory, students observe weak localization with spin effects at temperatures below about 14 $\mathrm{K}$. Above $14 \mathrm{~K}$, phonons destroy the spin effects, and spinless weak localization is observed.

\section{THEORY}

Weak localization is a correction to the Drude conductivity. Before we discuss the correction, we review the Drude prediction and its assumptions.

\section{A. Drude conductivity}

An electric field inside a normal metal will drive an electrical current. The current density $\mathbf{J}$ is related to the field $\mathbf{E}$ by

$$
\mathbf{J}=\sigma \mathbf{E},
$$

where $\sigma$ is the conductivity. An expression for the conductivity was derived by Drude, ${ }^{1}$ based on some simple assumptions about the microscopic properties of metals. If the current is carried by electrons, then the current density is

$$
\mathbf{J}=-n e\langle\mathbf{v}\rangle,
$$

where $n$ is the number of electrons per unit volume, $-e$ is the electric charge on a single electron, and $\langle\mathbf{v}\rangle$ is the average velocity of the electrons.

To calculate the average velocity, we assume that the electrons move ballistically until they collide with something. The electric field provides a force on the electrons given by $-e \mathbf{E}$, and the equation of motion is then

$$
m \frac{d \mathbf{v}}{d t}=-e \mathbf{E} .
$$

If we know an electron's velocity at time $t=0$, we can calculate it at a later time $t$, provided it does not have a collision.

$$
\mathbf{v}(t)=\mathbf{v}(0)-\frac{e \mathbf{E} t}{m} .
$$

For this particular electron, we assume that just before $t=0$ it had a collision with something inside the metal (an impurity or phonon, for example). Moreover, we assume that, just after the collision, the velocity of the electron is completely random, which implies that 


$$
\langle\mathbf{v}(0)\rangle=0
$$

With the assumption of isotropic scattering, the average velocity of all the electrons inside a metal is easy to calculate. We have

$$
\langle\mathbf{v}\rangle=-\frac{e \mathbf{E} \tau}{m},
$$

where $\tau$ is the average time since the last scattering event. This ensemble average is independent of the time when we take the snapshot and gives

$$
\sigma_{D}=\frac{n e^{2} \tau}{m} .
$$

Equation (7) is known as the Drude conductivity. It assumes that an electron's velocity is completely randomized after a scattering event. That is, the electron has no memory of its previous state after it suffers a collision. As we shall see, this assumption is good at room temperatures, but it breaks down at very low temperatures. The cause of this breakdown is the wave nature of the electron, which does not play a role in the derivation of the Drude conductivity. The resulting change in the conductivity is one of the few macroscopically observable consequences of the wave-particle duality of matter.

\section{B. Coherent backscattering}

When we calculated the Drude conductivity, we assumed the electrons were point particles, obeying the laws of classical physics. In reality electrons have associated wave functions. When the wave that describes an electron scatters off an obstacle, it produces partial waves that emanate from the obstacle, much like ripples in a pond produced by a wave when it hits a stationary reed sticking out of the surface. These partial waves go on to strike other obstacles, impurities, or defects in the case of a metal, and produce more partial waves. All of these partial waves add up to produce a complicated diffraction or interference pattern. For the most part, the phase between any two partial waves is random, and the partial waves add incoherently on the average (see Fig. 1). However, there is one direction in which the partial waves will always add up in phase. This direction is opposite to the initial wave, because, for every path that takes a partial wave back to its origin, there is a complementary path with the same length that takes the same route, but in the opposite direction. The two partial waves that take these complementary paths will always add coherently (see Fig. 2). The sum of all these complementary waves gives a slightly stronger wave going backward relative to the initial direction, which corresponds to an enhanced probability of backscattering, which in turn reduces the conductivity below the Drude prediction.

If the metal is infinitely large and the electrons can maintain phase coherence over infinitely long complimentary paths, then the backscattering effect dominates the dynamics of the electrons, and the conductivity is completely suppressed. In this case, the metal becomes an insulator, and the electrons are trapped, or localized, by the disordered scattering centers. For samples with finite size, or more commonly finite coherence lengths, the backscattering gives rise to a

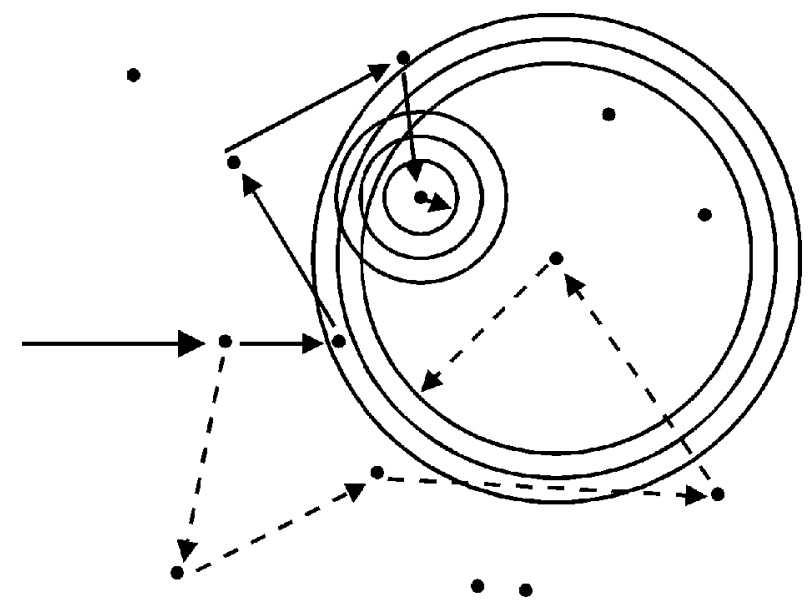

Fig. 1. Most partial waves have random relative phases, and add incoherently on average. In this figure, two scattering paths are considered, one shown by a solid line and one by a dashed line. Notice that the wave fronts for both scattering paths are separated by the same radial length. This separation is the Fermi wavelength, $\lambda_{F}$. For these randomly scattered partial waves, the wave fronts do not align, so on average there will be no net interference.

small correction to the Drude conductivity. This small correction should be called "prelocalization," but it is commonly referred to as "weak localization."

Coherent backscattering can occur only if the phase of each partial wave is preserved as it goes around its path. At high temperatures, where most scattering events are off phonons, coherent backscattering cannot occur. A magnetic field also can introduce a phase difference between the complementary paths, destroying the coherent backscattering and any correction to the Drude conductivity it produces. The resulting dependence of the conductivity on temperature or applied magnetic field is very small even at liquid helium temperatures, but it can be observed experimentally by employing a few basic low-noise techniques. In the experiment

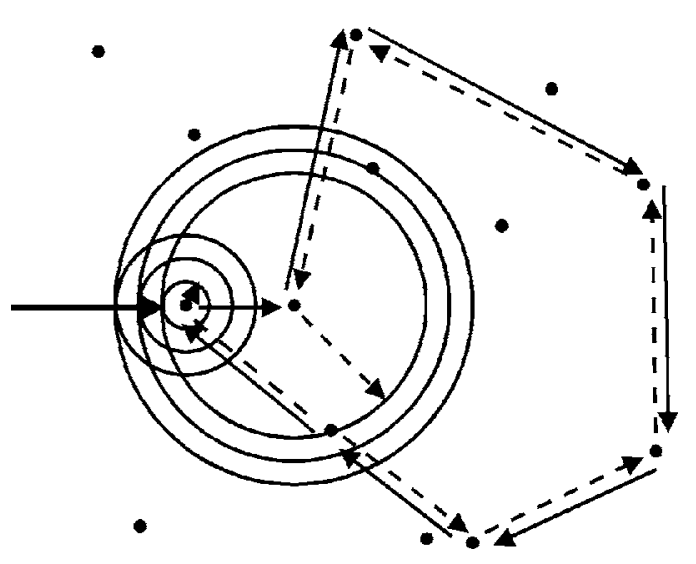

Fig. 2. Each partial wave that returns to the origin has another partial wave with which it is in phase in the backscatter direction. Again, we consider two partially scattered waves. Unlike Fig. 1, the two paths are the same except that they are traversed in opposite order. That is, if we apply time reversal to one path, we obtain the second path. This similarity, and the fact that their net scattering direction is in the backscattering direction, causes the wave fronts of the two paths to align on average, and there is a net interference effect. This interference means on average the backscatter probability is modified from the predictions of classical physics. 


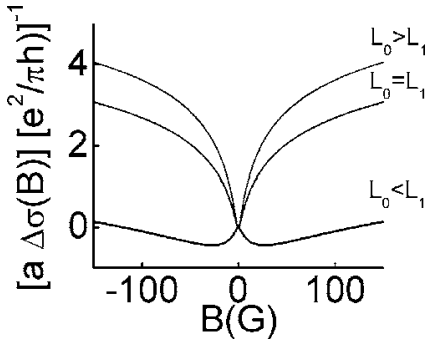

Fig. 3. The weak localization magnetoconductance at a fixed temperature. The different curves represent different relative contributions from spin effects.

we will describe in Sec. III, we discuss some basic low-noise and small-signal-detection methods that are commonly used.

\section{Weak localization}

A quantitative derivation of weak localization requires the use of quantum many-body theory and the quantum field theory techniques that go with it. Such a derivation is beyond the scope of this paper, and we will give the result without derivation. See Refs. 2 or 3 for a detailed treatment of the theory of weak localization.

The change in the conductance of a sample when a magnetic field is applied is called the magnetoconductance. This magnetoconductance is easiest to observe in a twodimensional sample, where we can apply a magnetic field that is perpendicular to the sample and thus perpendicular to all of the complimentary, closed-loop paths that give rise to the coherent backscattering. For a thin film (a nearly twodimensional sample), the magnetoconductance is

$$
\begin{aligned}
a \Delta \sigma(B)= & \frac{e^{2}}{\pi h}\left\{\frac{3}{2}\left[\psi\left(\frac{1}{2}+\frac{\hbar c}{4 e} \frac{1}{L_{1}^{2} B}\right)-\ln \left(\frac{\hbar c}{4 e} \frac{1}{L_{1}^{2} B}\right)\right]\right. \\
& \left.-\frac{1}{2}\left[\psi\left(\frac{1}{2}+\frac{\hbar c}{4 e} \frac{1}{L_{0}^{2} B}\right)-\ln \left(\frac{\hbar c}{4 e} \frac{1}{L_{0}^{2} B}\right)\right]\right\},
\end{aligned}
$$

where $a$ is the thickness of the film, $\Delta \sigma(B) \equiv \sigma(B)-\sigma(0)$, and $\psi$ is the digamma function, defined in terms of the ordinary gamma function $\Gamma(x)$ as

$$
\psi(x)=\frac{d}{d x} \ln \Gamma(x)=\frac{1}{\Gamma(x)} \frac{d \Gamma(x)}{d x} .
$$

A plot of Eq. (8) is shown in Fig. 3. The curves in Fig. 3 are plotted at fixed temperature, but they have varying contributions of effects due to spin, which is accomplished by varying the ratio of $L_{0}$ and $L_{1}$.

The dephasing lengths $L_{0}$ and $L_{1}$ are combinations of the average distance that an electron can diffuse before colliding with a phonon, $L_{\phi}$, and the average distance an electron can diffuse before it becomes dephased by spin-orbit, $L_{\mathrm{so}}$, or spin-flip, $L_{\mathrm{sf}}$, interactions with the scatterers,

$$
\begin{aligned}
& \frac{1}{L_{0}^{2}}=\left(\frac{1}{L_{\phi}^{2}}+\frac{2}{3 L_{\mathrm{sf}}^{2}}\right)+\frac{4}{3 L_{\mathrm{sf}}^{2}}, \\
& \frac{1}{L_{1}^{2}}=\left(\frac{1}{L_{\phi}^{2}}+\frac{2}{3 L_{\mathrm{sf}}^{2}}\right)+\frac{4}{3 L_{\mathrm{so}}^{2}} .
\end{aligned}
$$

A film is considered thin, or quasi-two-dimensional, if it is much thinner than the typical dephasing lengths, $a \ll L_{0}, L_{1}$.

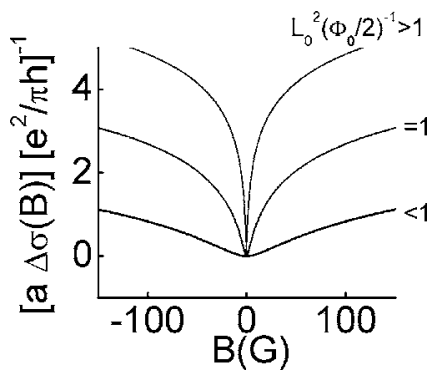

Fig. 4. The weak localization magnetoconductance in a sample where spin effects are negligible, at several temperatures $\left(\Phi_{0}=\hbar c / 4 e\right)$.

If spin effects are negligible, $L_{0}=L_{1}=L_{\phi}$, and the weak localization magnetoconductance is particularly simple,

$$
a \Delta \sigma(B)=\frac{e^{2}}{\pi h}\left[\psi\left(\frac{1}{2}+\frac{\hbar c}{4 e} \frac{1}{L_{\phi}^{2} B}\right)-\ln \left(\frac{\hbar c}{4 e} \frac{1}{L_{\phi}^{2} B}\right)\right] .
$$

A plot of Eq. (12) at various temperatures is shown in Fig. 4, where $L_{0}$ is varied by changing the temperature, because the average inelastic dephasing length depends on temperature.

For most conductors, the spin-flip interaction is negligible $\left(L_{\mathrm{sf}} \gg L_{\mathrm{so}}, L_{\phi}\right)$, and the terms proportional to $1 / L_{\mathrm{sf}}^{2}$ can be safely neglected. The spin-orbit term, however, scales with the atomic number of the metal, $1 / L_{\mathrm{so}}^{2} \sim Z^{4}$. For light metals, such as lithium or magnesium, spin-orbit effects are negligible. For heavier metals, such as silver or gold, spin-orbit effects can dramatically alter the weak localization magnetoconductance signature.

In comparison to the Drude conductivity, the weak localization correction is very small. At most, the fractional correction to the conductivity, $\delta \sigma \equiv \sigma(0)-\sigma_{D}$, is of the order

$$
\left|\frac{\delta \sigma}{\sigma}\right| \leqslant \frac{e^{2}}{a \pi h} \frac{m}{n e^{2} \tau} .
$$

For a typical metal film with a thickness of $a=100 \AA$, this ratio is

$$
\left|\frac{\delta \sigma}{\sigma}\right| \leqslant 10^{-4} .
$$

To measure magnetoconductance with an accuracy of $1 \%$, we need to resolve changes in the total conductivity on the order of parts per million. This resolution demands low-noise and small-signal detection methods. Teaching these methods is one of the purposes of this laboratory.

\section{EXPERIMENTAL APPARATUS}

\section{A. Samples and their preparation}

The first activity that our students do is prepare their thin film Ag samples. To make this preparation easy, we have assembled a rugged evaporative deposition system in our laboratory. Evaporative deposition is simple enough so that most laboratories are in possession of the equipment needed to set up an evaporative deposition system. The basic idea is to make a tightly sealed chamber and evacuate it using vacuum pumps, so that the pressure is low enough for air to be in the molecular flow regime; namely, we want the mean free path to be longer than the dimensions of the vacuum chamber. Under these conditions, evaporative deposition can be performed by melting a Ag pellet. The resulting gas of $\mathrm{Ag}$ 
atoms will travel to the walls of the chamber with a low probability of hitting other molecules. If a substrate is placed on one of the walls, it is possible to deposit a thin film of $\mathrm{Ag}$ onto this substrate. To satisfy the criteria of making the film quasi-two-dimensional, that is, $a \ll L_{\phi}$, the films need to be between 60-200 $\AA$ thick. To make sure that the samples are that thin, our students use a commercially available thickness gauge $^{4}$ to monitor the thickness of their films.

We use a commercially available liquid ${ }^{4} \mathrm{He}$ dewar from Quantum Design, ${ }^{5}$ which comes with a 5 Tesla superconducting magnet, to cool the sample and make measurements in a magnetic field. This apparatus is not the only option. The experiment can be done just as easily with an inexpensive dipping probe, inserted directly into a liquid ${ }^{4} \mathrm{He}$ storage dewar. In fact, such a system would probably be preferable. The computer-controlled Quantum Design cryostat can sometimes obscure the physics of the experiment, and the central focus of the laboratory should be on the physics of electron transport in mesoscopic systems and techniques for low-level signal detection.

To prepare the samples for measurement, students apply contacts to the film, and make electrical measurements on the film when it is at liquid ${ }^{4} \mathrm{He}$ temperatures $(T \geqslant 2 \mathrm{~K})$. For our setup, students make contact from the sample to a resistivity sample stage, called a puck. ${ }^{6}$ The contact is made using silver paste and gold wires. Once the contacts are made, the sample stage is loaded into the cold dewar. We then provide a breakout box that allows connections to be made to the resistivity puck from inside the dewar.

\section{B. Detection of magnetoresistance}

To relate the observable resistance change to the prediction for the change in the conductivity, we start with the relation between resistance and conductivity for a film of thickness $a$, length $l$, and width $w$,

$$
R=\frac{1}{\sigma a w} .
$$

The change in the resistance due to a change in the conductivity is

$$
\Delta R=-\frac{\Delta \sigma}{\sigma^{2}} \frac{l}{a w}=-\frac{a \Delta \sigma}{a \sigma} R=-a \Delta \sigma R_{\Lambda} R,
$$

where $1 / a \sigma \equiv R_{\Lambda}$ is the resistance of a square film. Equations (8) and (12) give predictions for $a \Delta \sigma(B)$, which we will compare with measurements of $-\Delta R / R R_{\Lambda}$.

To obtain $R$ and $R_{\Lambda}$, we pass a small excitation current through the sample and measure the resulting voltage across the sample. This measurement gives the total resistance of the sample $R(0)$, from which we can calculate $R_{\Lambda}=R(0) w / l$, provided we know the sample's length $l$ and width $w$.

Measuring the magnetoresistance $\Delta R(B)$ is done in a similar way, but because $\Delta R(B)$ is so much smaller than the background signal $R(0)$, we need to use an experimental trick, nulling. What we are really measuring is the voltage across the sample produced by the excitation current $i$, or $V(H)=i R(B)$. If we can produce a reference voltage that is equal to the zero-field voltage across the sample, $V_{0}=i R(0)$, then we can subtract this value from the actual voltage across the sample to obtain $\Delta R(B)$,

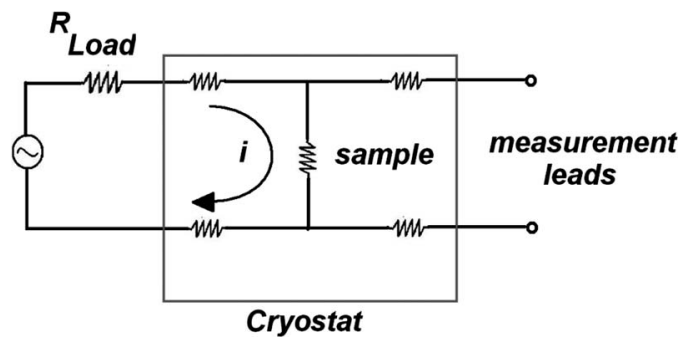

Fig. 5. A four-wire arrangement. No current flows through the contact resistances, so when we measure a voltage, it is due to the sample only.

$$
V(H)-V_{0}=i R(B)-i R(0)=i \Delta R(B) .
$$

This very small signal can then be amplified and examined in detail as a function of the magnetic field $B$.

The process of nulling will work only if we can produce a small voltage $V_{0}$ that is independent of the magnetic field. Fortunately, this is easy to do using a passive, adjustable, low noise voltage divider. We used a Dekatran DT72A tunable voltage divider, ${ }^{7}$ driven by the same voltage that produced the excitation current for the sample.

In both the $R_{\Lambda}$ and $\Delta R(B)$ measurements, it is important not to use the same set of wires to carry the excitation current and to measure the resulting voltage across the sample. Long wires leading into the cryostat may have resistances of their own, which, if their leads carry current, will produce voltages that have nothing to do with the sample. To measure only the voltage produced by the sample, we employ a fourwire geometry as shown in Fig. 5. For this four-wire technique to be effective, no current must be allowed to flow along the measurement leads. If it did, a spurious voltage would be produced from the resistances in the measurement leads, which would contaminate the measurement. Such a condition can result, for example, from the measurement device and the excitation source sharing a common ground, as shown in Fig. 6. This condition is known as a ground loop, and care is needed to avoid it.

All of these measurements are performed at audio frequencies using a lock-in amplifier, to which the students have been introduced in a previous lab. ${ }^{8}$ We add to their training with a lock-in by encouraging them to analyze the noise in each component of their apparatus and in the apparatus as a whole. This analysis is easily done with a lock-in by terminating the input of a device with a $50 \Omega$ terminator, and then measuring the noise of the output signal on the lock-in. Students can then determine which component is setting their noise floor; our students find the pre-amplifier sets their noise floor.

Lock-in detection, nulling, four-wire measurements, and ground loops are all essential topics with which modern,

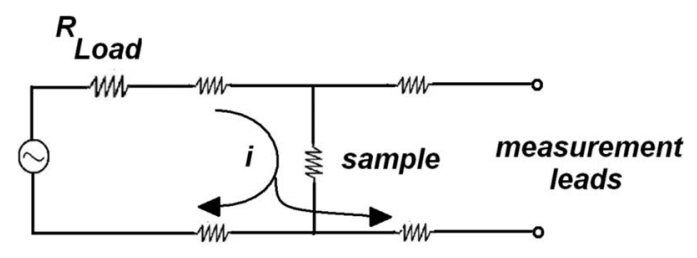

Fig. 6. An example of how a ground loop may develop in a four-wire measurement. The lock-in inputs we use are floated to prevent this condition. 


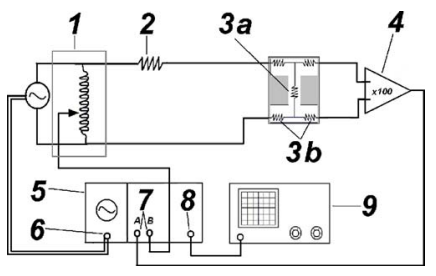

Fig. 7. The setup used to measure weak localization. It is a four-wire resistance bridge that utilizes a lock-in, a preamplifier, and a decade transformer to resolve the magnetoresistance, (1) decade transformer, (2) $35 \mathrm{k} \Omega$ resistor, (3a) sample resistance, (3b) contact resistances, (4) SR60 preamplifier, (5) SR830 lock-in amplifier, (6) internal lock-in reference, (7) lock-in inputs, (8) $10 \mathrm{~V}$ proportional output, (9) oscilloscope.

condensed matter experimentalists are familiar, and these labs provide students with a thorough, quantitative, foundation in each. A brief schematic of our entire apparatus for performing these functions is shown in Fig. 7.

\section{TYPICAL RESULTS}

By using the techniques we have described, our students found results consistent with weak localization. Figure 8 shows typical magnetoresistive data and fits to the data. The results clearly show weak localization with spin effects at low temperatures $(T \leqslant 14 \mathrm{~K})$, as evidenced by the initially positive magnetoresistance at low magnetic fields followed by negative magnetoresistance at higher field values. This feature can be completely described using Eq. (16) to convert Eq. (8) to a magnetoresistance prediction, and then fitting the data. The switch from positive to negative magnetoresistance represents a competition between the positive magnetoresistance due to spin effects, and the loss of coherence due to the magnetic field, which produces a negative magnetoresistive effect. At high enough magnetic fields, the loss of coherence due to the magnetic field dominates the positive magnetoresistance, and the sign of the magnetoresistance changes.

At $T \geqslant 14 \mathrm{~K}$, Eq. (12) can be used to describe the magnetoresistance [after being converted using Eq. (16)]. The fact that we can use Eq. (12) instead of Eq. (8) reflects the fact that the average spin-orbit scattering length is much longer

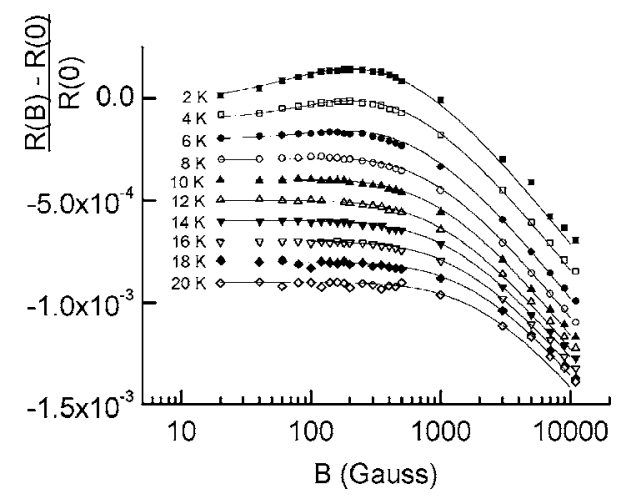

Fig. 8. Typical data for the magnetoresistance versus field. The field scale is a log scale. Weak localization, with spin effects, is evident from $2 \mathrm{~K}$ to about $14 \mathrm{~K}$, as a positive magnetoresistance at low fields and a negative magnetoresistance at larger fields. The data at temperatures above $2 \mathrm{~K}$ is offset for clarity; the magnetoresistance goes to 0 when $B=0$. The lines are fits to Eq. (16).

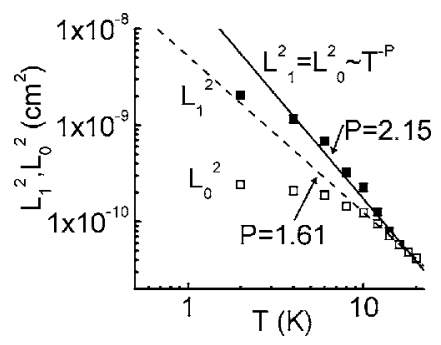

Fig. 9. Square of the coherence length versus temperature. The linear portion above $14 \mathrm{~K}$ has a slope between -1.61 and -2.15 . If two-dimensional thermal phonons dominate inelastic scattering, the slope should be 2 .

than the average phonon scattering length for $T \geqslant 14 \mathrm{~K}$. That is, when $L_{\Phi} \ll L_{\mathrm{so}}, L_{0}=L_{1}=L_{\phi}$ in Eq. (8), and we obtain Eq. (12). For $T \geqslant 14 \mathrm{~K}$, electron-phonon interactions are more important than spin-orbit interactions. This statement is physically equivalent to saying that $L_{\Phi} \ll L_{\mathrm{so}}$. So for $T$ $\geqslant 14 \mathrm{~K}$, spin interactions, due to spin-orbit scattering, are negligible, and we observe weak localization without spin effects.

To fit the data in Fig. 8 for all temperatures, the students performed a two parameter fit of Eq. (8) converted into the magnetoresistive form by Eq. (16). This fit requires the parameters, $L_{1}$ and $L_{0}$, to be adjusted until a good fit is found. We encouraged the students to fit the equations visually until the fits were close, which required the students to think about the relevant length scales of $L_{1}$ and $L_{0}$ and what they mean. $L_{0}$ is the average length that an electron travels before undergoing an inelastic scattering event with a phonon. The parameter $L_{1}$ describes whether electrons scatter more frequently from phonons or from disorder, via spin-orbit scattering. The student values of $L_{0}$ and $L_{1}$ for the fits in Fig. 8 are shown in Fig. 9.

The typical length scale for $L_{1}$ and $L_{0}$ is about $0.5 \mu \mathrm{m}$, and at $T \leqslant 14 \mathrm{~K}, L_{1}>L_{0}$. The fact that $L_{1}>L_{0}$ indicates that spin-orbit scattering is more frequent than phonon scattering at low temperatures, as we expect, and weak localization, with spin effects, is observed.

Above $14 \mathrm{~K}$ (as shown in Fig. 9), $L_{1} \cong L_{0}$, and the log-log plot of $L_{0}^{2}$ and $L_{1}^{2}$ versus temperature has a slope between -1.61 and -2.15 . In a paper with results similar to ours, Gershenzon ${ }^{9}$ pointed out that the slope of $L_{1}^{2}$ and $L_{0}^{2}$ versus temperature should be equal to -2 if we expect twodimensional thermal phonons to be the source of inelastic scattering. So above $14 \mathrm{~K}$, our students are likely observing the excitation of two-dimensional thermal phonon modes, which causes the spin effects of weak localization to become less important.

\section{SUMMARY}

Our experiment on weak localization introduces many of the experimental techniques that are used in contemporary research. Students learn to make their own samples, make electrical contacts to them, cool them, and then make measurements. In addition, the resistance bridge technique is a common technique to observe small signals on top of a large background signal.

The typical student results indicate that our technique is able to observe the predicted macroscopic expression of 
weak localization through the magnetoresistance of $\mathrm{Ag}$ thin films. Their results also show that the effect is observable at easily accessible temperatures.

\section{ACKNOWLEDGMENT}

This work was supported by the National Science Foundation under Grant No. DUE-0088658.

\footnotetext{
${ }^{\text {a)}}$ Electronic mail: beyer@its.caltech.edu

${ }^{1}$ Paul Drude, "Zur elektronentheori der metalles 1 Teil," Ann. Phys. (Leipzig) 1, 566-613 (1900); "Zur elektronentheori der metalles 2 Teil, galvanomagnetische und thermomagnetisch effecte," ibid. 3, 369-402 (1900).

${ }^{2}$ Gerd Bergmann, "Weak localization in thin films: A time-of-flight experi-
}

ment with conduction electrons," Phys. Rep. 107, 1-58 (1984).

${ }^{3}$ Sudip Chakravarty and Albert Schmid, "Weak localization: The quasiclassical theory of electrons in a random potential," Phys. Rep. 140, 193-236 (1986)

${ }^{4}$ XTC/C: Thin Film Deposition Controller. Infinicon, Two Technology Place, East Syracuse, NY 13057-9741.

${ }^{5}$ Physical Property Measurement System. Quantum Design, San Diego, CA 92121-3733, <http://www.qdusa.com/>

${ }^{6} \mathrm{DC}$ resistivity puck. Quantum Design.

${ }^{7}$ Dekatran DT72A. Tegam, Ten Tegam Way, Geneva, OH 44041, < http:// www.tegam.com/>

${ }^{8}$ K. G. Libbrecht, E. D. Black, and C. M. Hirata, "A basic lock-in amplifier experiment for the undergraduate laboratory," Am. J. Phys. 71 12081213 (2003)

${ }^{9}$ M. E. Gershenzon, V. N. Gubankov, and Yu. E. Zhuravlev, "Weak' localization and electron scattering in silver thin films," JETP Lett. 35, 576-580 (1982). 